\title{
Energy Storage Requirements for PV Power Ramp Rate Control in Northern Europe
}

\author{
Julius Schnabel and Seppo Valkealahti \\ Department of Electrical Engineering, Tampere University of Technology, P.O. Box 692, 33101 Tampere, Finland \\ Correspondence should be addressed to Seppo Valkealahti; seppo.valkealahti@tut.fi
}

Received 1 April 2016; Revised 24 May 2016; Accepted 2 June 2016

Academic Editor: Prakash Basnyat

Copyright (C) 2016 J. Schnabel and S. Valkealahti. This is an open access article distributed under the Creative Commons Attribution License, which permits unrestricted use, distribution, and reproduction in any medium, provided the original work is properly cited.

\begin{abstract}
Photovoltaic (PV) generators suffer from fluctuating output power due to the highly fluctuating primary energy source. With significant PV penetration, these fluctuations can lead to power system instability and power quality problems. The use of energy storage systems as fluctuation compensators has been proposed as means to mitigate these problems. In this paper, the behavior of PV power fluctuations in Northern European climatic conditions and requirements for sizing the energy storage systems to compensate them have been investigated and compared to similar studies done in Southern European climate. These investigations have been performed through simulations that utilize measurements from the Tampere University of Technology solar PV power station research plant in Finland. An enhanced energy storage charging control strategy has been developed and tested. Energy storage capacity, power, and cycling requirements have been derived for different PV generator sizes and power ramp rate requirements. The developed control strategy leads to lesser performance requirements for the energy storage systems compared to the methods presented earlier. Further, some differences on the operation of PV generators in Northern and Southern European climates have been detected.
\end{abstract}

\section{Introduction}

Solar radiation fluctuates in annual, daily, and down-tosecond timescale. Fast fluctuations are dominantly due to shading caused by moving clouds. Accordingly, the power behavior of photovoltaic generators (PVGs) is known to be rapidly and intensively fluctuating even for large generators $[1,2]$. Fast power fluctuations can lead to power quality problems in the power system, and feeding them into a power system in large quantities can also cause serious stability issues. Thus, concerned power system operators are demanding regulation of $\mathrm{PV}$ power variability in the form of ramp rate (RR) limitation [3].

Energy storage systems (ESS) have been proposed as the solution for compensating the variability of PV power and other variable energy sources [4-8]. The key issue in utilizing ESS for compensating PV power fluctuations is the sizing of the storage unit to minimize the obvious addition of economic costs. Essential technical sizing variables are the required energy capacity, maximum power output, and the charge-discharge-cycling induced degradation.
Minimization of these variables reduces the added economic burden for the PV power producer. Studies have shown how the right selection of the ESS control strategy can reduce the amount of required energy capacity [9-11] and application induced degradation [12]. Although some of these studies have varied the studied location of the PV system, none have yet examined PV ramp rate limiting in Northern European climate and the effects of such climate on ESS sizing.

In [9], the authors reasoned that both the maximum required ESS power and energy capacity could be analytically derived from a so-called worst fluctuation model (WFM). The model relies on the idea that with proper control the maximum energy surplus or deficiency would be dictated by PVG power fluctuations with the worst possible characteristics. These characteristics were selected to be a fast transition from 100 to $10 \%$ of the nominal PVG output power, or vice versa, while the fall or rise of the compensated grid feedin power would be uninterrupted. The model was validated by comparing the calculated requirements with simulations of virtual storage systems compensating fluctuations of measured PVG power over a full year. The comparison showed 
distinct overlap between the calculated requirements and simulation results. However, all the data used in the study originated from a Southern European climate. Intuition says that this climate can differ from other climates, especially those in the Northern latitudes. Moreover, the WFM is a kind of worst-case method for ESS sizing and there is obvious need for more economical control strategies.

In this paper, PV power fluctuations have been studied by utilizing the irradiance measurements of the Tampere University of Technology (TUT) solar PV power station research plant located in Tampere, Finland [13]. Irradiance measurements and existing models for spatial smoothing of PV generator power fluctuations [12, 14] have been utilized to conduct generic investigations without being limited to detailed system specifications. Compensation of the analyzed PV power fluctuations has then been simulated with an arbitrary virtual ESS, for which an enhanced charging control strategy has been developed, tested, and applied to obtain optimal performance requirements for the ESS. The behavior of the ESS has been monitored against two years of measurement data, and conclusions have been drawn about the sizing requirements and degradation of the ESS. The results demonstrate that the introduced ESS control strategy performs better than the earlier applied strategies [9-11], and also some geographical dependency of $\mathrm{PV}$ power fluctuations as well as optimal ESS sizing appears.

\section{Measurement Data}

The data used in this paper was obtained from the TUT solar PV power station research plant located in Tampere, Finland. The plant contains a $13 \mathrm{kWp}$ PV generator consisting of various $\mathrm{PV}$ module string formations. All modules are facing closely due south and tilted $45^{\circ}$ from the planar roof plane. The PV module strings are attached with a network of 24 pairs of irradiance (Kipp \& Zonen SP Lite2) and temperature (PT100) sensors. The irradiance sensors are plane of array sensors mounted next to PV modules with the same $45^{\circ}$ tilt angle. The temperature sensors are mounted on the back plates of the same modules. In addition, comprehensive climatic measurements have been conducted. Synchronized measurements have been recorded with a $10 \mathrm{~Hz}$ sampling frequency continuously since June 2011 [13].

All measurements used in this paper have been upscaled to a sufficient $1 \mathrm{~Hz}$ sampling frequency. Measurement noise has been filtered from all datasets by applying a 5-second moving average. Most of the analyses in this paper rely on measurements from the 2012 leap year. The dataset of 2012 has 17 days with insufficient data, leaving 349 days (95\%) for examination. Cross-check of the results has been done with a dataset from 2014, which has 298 viable days (82\%). Missing data is due to update and maintenance of the research plant. Both datasets contain extensive amounts of clear sky, overcast, and half cloudy days for diurnal variability and all months are sufficiently represented for seasonal diversity.

An example of a recorded daily irradiance profile is shown in Figure 1, which demonstrates the frequency and severity of irradiance fluctuations. These fluctuations are translated

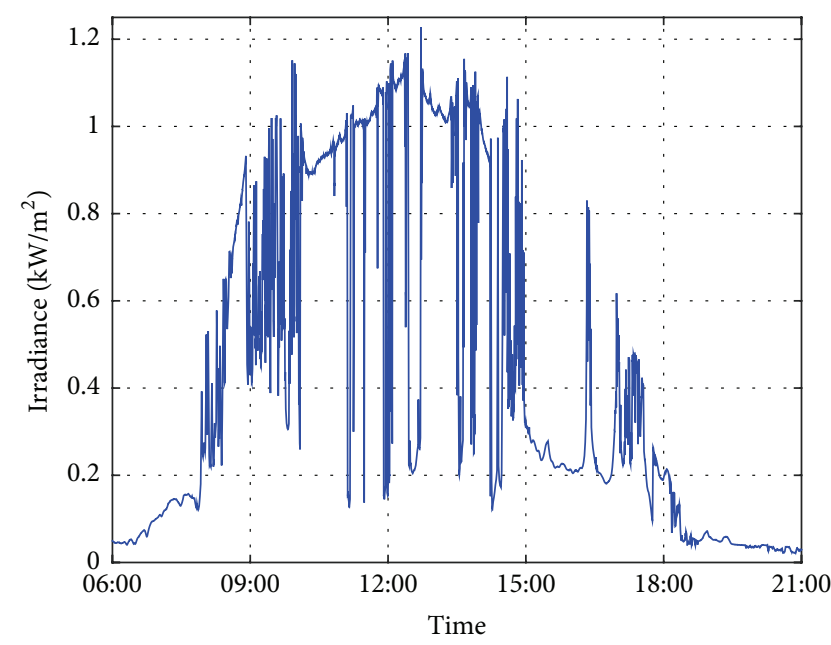

FIGURE 1: Solar irradiance received by PV module of the TUT solar PV power station research plant measured on 26.09.2012.

into the power behavior of any PV generator located in the same area using existing models for spatial smoothing of PV generator power fluctuations $[12,14]$. The ramp rates of these irradiance fluctuations give a good qualitative metric for variability. For example, during the day presented in Figure 1 the observed 1-second irradiance ramps exceed the commonly applied ramp rate limit of $10 \% /$ min for a total time of 2 hours and 33 minutes and practically all visible fluctuations exceeded the limit. This demonstrates clearly why an increasing number of power system operators are concerned about the growing penetration of grid-connected $\mathrm{PV}$ systems and consider imposing ramp rate limits for PV power generation within their grid domains.

\section{PV Generator Power Model}

In order to achieve general and widely applicable results, the ESS simulations need to be based on data with minimum amount of restrictions and preconditions. In case of real PV power plants, the output power always depends on the power plant layout and the used PV arrays topologies, inverters, maximum power point tracking algorithms, and so forth. Therefore, the used data should rather be an irradiance based approximation of PVG power for obtaining technology independent results. Different methods exist to model PV generator power directly based on irradiance measurements [12].

The model used in this paper relies on the direct proportionality of PVG power to incident irradiance. Spatial smoothing of irradiance over large areas during irradiance transitions [1], which are associated with corresponding PV generators, is taken into account using the low-pass filter method presented in [14]. The method uses a single irradiance point measurement and PVG area as inputs. It is assumed in the method that the PVG covers a symmetric square shaped area. The time series of spatial irradiance $G_{s}(t)$ 
can be obtained by applying the first-order low-pass filter transformation as follows:

$$
G_{s}(t)=\frac{G(t)}{\tau s+1},
$$

where $G(t)$ is the measured irradiance time series of a point sensor, $s$ is the Laplace transform variable, and $t$ is time. For the low-pass filter time constant, the following approximation is applied:

$$
\tau=\frac{\sqrt{A}}{2 \pi \cdot a}
$$

where constant $a=2 \mathrm{Hzm}$ is obtained from a fit to the cutoff frequencies of power spectra of various PV power plants plotted as a function of plant area $A$ [14]. $A$ is the effective area of the PV power plant realizing the spatial smoothing in the transfer function; that is, the time constant is directly proportional to the effective dimension of the power plant. Hereby, spatial smoothing increases with increasing time constant.

In addition to spatial smoothing of PV power fluctuations, the model takes into account the PV module temperature, which can cause notable offset of power with respect to standard operating conditions. A temperature correction is applied based on measured polycrystalline silicon PV module temperatures of the TUT solar PV power station research plant with a power decrease of $0.5 \% /{ }^{\circ} \mathrm{C}$ as follows $[13,15]$ :

$$
P_{\text {mod }}(t)=\frac{G_{s}(t)}{G_{\mathrm{STC}}} \cdot\left[1-0.005 \frac{1}{{ }^{\circ} \mathrm{C}} \cdot\left(T(t)-T_{\mathrm{STC}}\right)\right],
$$

where $G_{\text {STC }}$ and $T_{\text {STC }}$ are the irradiance and PV module temperature in standard test conditions (STC), respectively, and $T$ is the measured PV module temperature. Note that (3) gives the modelled power $P_{\bmod }$ in per-unit convention, which is easily scalable to relevant PV generator sizes.

In this paper, the spatial irradiance model is used to approximate the power behavior of virtual PVGs of different sizes as if they were operating in the TUT solar PV power station research plant area. Further, the results of the ESS simulations are compared to findings of similar studies presented in [9]. For the sake of proper comparison, two PVG sizes of 0.55 and 2.2 MWp were selected for examination with corresponding PV plants characteristics as in the reference power plants located in Southern Europe [9]. An example on the behavior of the spatial irradiance smoothing model can be seen in Figure 2 for the selected two PVG sizes.

\section{Energy Storage System Simulations}

Simulations of the energy storage system operation were carried out using the presented PV generator power model and measurements from the TUT solar PV power station research plant. The ESS was assumed to be an arbitrary virtual system with no predefined preconditions and limitations on power or energy. As mentioned earlier, two different PV generator sizes of 0.55 and $2.2 \mathrm{MWp}$ nominal output power were studied. Their respected square shaped areas were

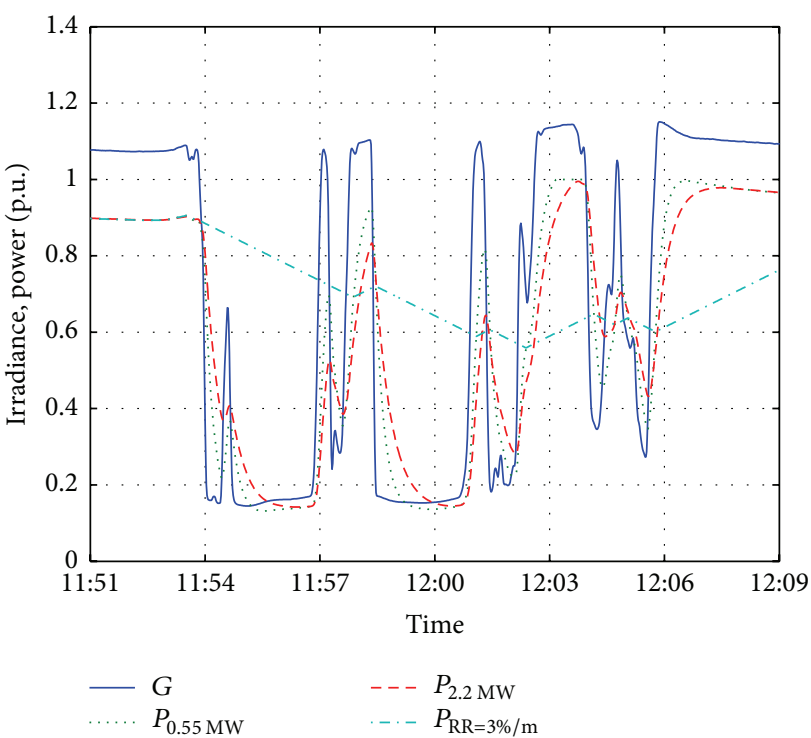

FIGURE 2: Measured point irradiance, simulated power of 0.55 and 2.2 MWp PV generators, and the grid feed-in power of the $0.55 \mathrm{MWp}$ PV generator complying RR limit of $3 \% /$ min during 18 minutes on 29.06.2012. Irradiance has been normalized to STC irradiance and power to the corresponding PV generator nominal power.

$25000 \mathrm{~m}^{2}$ and $100000 \mathrm{~m}^{2}$, when a ground cover ratio of 0.16 was assumed.

Different ESS control principles exist and their effects on the ESS sizing are notable. The ESS control principle used in this paper is a simple RR limiting control, because it is straightforward to apply and easily comparable and has been applied in practice by electric power companies. It provides a simple way to understand the consequences of different ramp rate limiting requirements and also demonstrates the importance of climatic effects on PV power production.

Compensation of grid feed-in power fluctuations was realized in the simulations in a similar way as in [9]. The modelled PVG power $P_{\mathrm{PVG}}$ is fed through the ramp rate limiter to calculate the desired limit compliant grid feed-in power $P_{\text {grid }}$. The limiter simply forces the difference between the present and the next value of $P_{\text {grid }}$ to be within a specified RR limit. The amount of ESS power $P_{\text {ESS }}$ can then be determined as the difference between the desired grid feedin power and the PVG power. In this manner, the fluctuations of $P_{\mathrm{PVG}}$ are compensated by charging the ESS with the excess power during the upward ramps and discharging the ESS during the downward ramps. Note that in our notation the ESS power is positive when ESS is discharging and feeds power to the grid.

In addition to this basic procedure, the ESS utilizes a state of charge (SOC) control. This control actively balances the ESS energy $E_{\mathrm{ESS}}$ towards a reference value $E_{\mathrm{ESS} \text {,ref }}$ by charging or discharging the ESS to minimize the required energy storage capacity [9]. A control block diagram of the simulated system utilizing SOC control is displayed in Figure 3.

The SOC control is a feedback-loop, where the PVG power is reserved from the grid feed-in power to balance ESS energy. The amount of reserved power can be controlled 


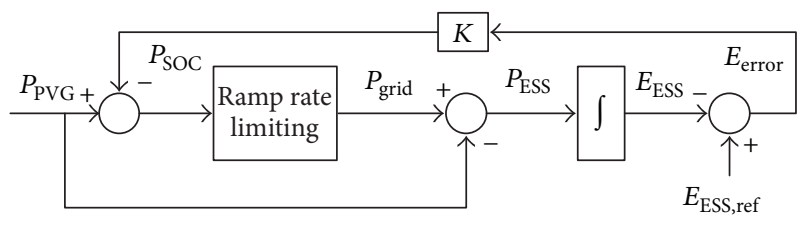

FIGURE 3: Block diagram of the used PV power ramp rate limiting procedure using an energy storage system equipped with state of charge control [9].

by setting an energy reference for the ESS and adjusting the proportional controller gain $K$. An optimum $K$ value was found to be $1.5 \cdot 10^{-3}$ for all the cases presented in this paper. The reference value for $E_{\text {ESS,ref }}$ was set to be $0 \mathrm{Wh}$ for convenience and deviations from this level were monitored to study the PV generator induced requirements for the ESS.

A noteworthy issue associated with SOC control is the possibility of producing an unintentional grid feed-in outage after a large downward fluctuation, while some PVG power due to diffuse radiation is still available. Many of the proposed control procedures in the literature seem to operate in such a way that they actually increase the overall power ramps in special circumstances and even cause grid feed-in power outages. This issue should be taken into account in the control of $P_{\text {grid }}$. The method used in this paper simply holds $P_{\text {grid }}$ at a constant level after a large downward ramp has reached the generated power $P_{\mathrm{PVG}}$ until the SOC control is ready to increase $P_{\text {grid }}$ again [15].

The simulated overall behavior of the RR limitation with ESS using the advanced SOC control strategy can be seen in Figure 4 , where the simulated $P_{\mathrm{PVG}}, P_{\text {grid }}, P_{\mathrm{ESS}}$, and $E_{\mathrm{ESS}}$ of the $0.55 \mathrm{MWp}$ PV generator are shown as a function of time for a day with some major fluctuations. The amount of $\mathrm{PV}$ generator power fluctuations fed into the grid is reduced due to ESS operation, and the remaining ramps have gentler slopes than in the original $P_{\mathrm{PVG}}$. In order to realize this, the ESS had to be able to provide the grid of at least 0.81 p.u. $(0.45 \mathrm{MW})$ peak power during that day in order to achieve full RR limit compliance.

The required storage of energy by the ESS during the selected day for compensating the fluctuations can be seen also in Figure 4. The negative values refer to discharged energy deficiency and positive values refer to charged energy surplus. The difference between the maximum and minimum energies determines the amount of required energy capacity $C_{\text {ESS }}$ of ESS, which was $0.16 \mathrm{~h}(88 \mathrm{kWh})$ during that single day. It is also seen in Figure 4 how the ESS eventually reaches the ESS reference value $E_{\mathrm{ESS}, \text { ref }}$ of $0 \mathrm{Wh}$ after fluctuations.

This is in place to shortly clarify the main differences between our approach for obtaining ESS requirements for Northern European climate and the approach behind the state-of-the-art results for Southern European climate [9], since we compare the results. The starting point difference is that we use solely irradiance measured with $10 \mathrm{~Hz}$ sampling frequency and obtain PVG power data by spatial smoothing [10], but 5-second PV power plant data has been utilized in [9]. Then, in [9] a sudden worst-case irradiance fluctuation from $100 \%$ to $10 \%$, or vice versa, is assumed followed with a corresponding exponential decay of PVG power. Instead, one-year irradiance based PVG power data is directly analyzed in our study without any further assumptions about the PVG power decay during the irradiance ramps. The exponentially decaying worst fluctuation model of PVG power applied in [9] seems to work as planned, since real PVG power ramps are much smoother. One more difference of principle between the approaches is that we apply ESS control strategy, which restores ESS back towards the reference charge level after power ramps (see Figures 6, 7, and 9).

\section{Energy Storage System Requirements}

The example provided in Figure 4 demonstrates how the ESS requirements are formed. In order to produce a more valid examination which takes into account seasonal behavior and diurnal differences, the same study was extended over the course of the full year of 2012. The effective energy capacity and maximum power and cycle counts of the simulated ESS were gathered and analyzed and finally requirements for ESS were deduced. Furthermore, the study was renewed with data from the year 2014 to verify that no significant anomalies were present in 2012.

In order to verify the correctness of our research approach and the feasibility of the developed ESS control strategy, results are compared to results obtained by applying the worst fluctuation model from corresponding PV power plants located in Southern Europe [9]. Since the authors are affiliated mainly with the University of Navarra in Spain, the data gathered from the reference site is titled as the UNav results. The measurement data used in this study obtained in the Northern European climate at the Tampere University of Technology is titled, in turn, as TUT results. Both results are presented as commensurately as possible to reveal the possible differences caused by the applied methods and different climatic conditions.

5.1. Energy Capacity Requirements. The effective energy capacity requirements of ESS $C_{\text {ESS }}$ for the two PV generator sizes are presented in Figure 5 as a function of applied RR limit both for the TUT and for the UNav results. The TUT capacities have been calculated using our enhanced ESS charging control strategy and the UNav capacities are based on the WFM charging control. Both TUT and UNav results show that the ESS capacity requirements are directly proportional to the PV generator size, since in both studies the normalized capacities of the two generator sizes are equal as a function applied RR limit. This finding is in line with earlier finding for PV generator sizes from $1 \mathrm{kWp}$ up to $100 \mathrm{MWp}$ [15]. The capacity requirement decreases considerably with decreasing power fluctuation control requirement (increasing RR limit) for both results. The ESS capacity requirement increases drastically if the required RR limit is decreased below $5 \% / \mathrm{min}$. It is evident that the ESS capacity requirement decreases considerably, if high RR limits are sufficient to ensure power quality and balance in the grid. 


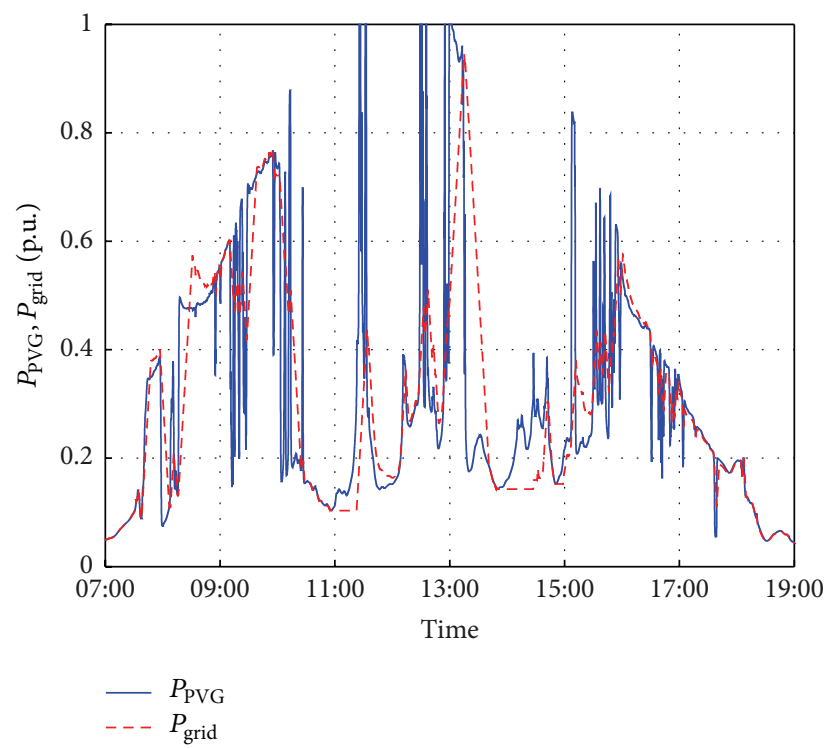

(a)

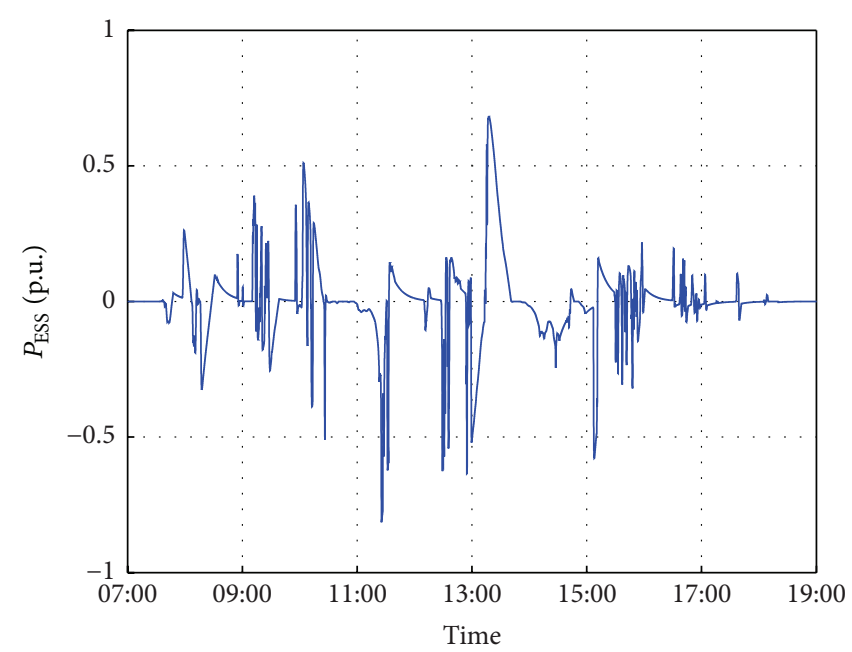

(b)

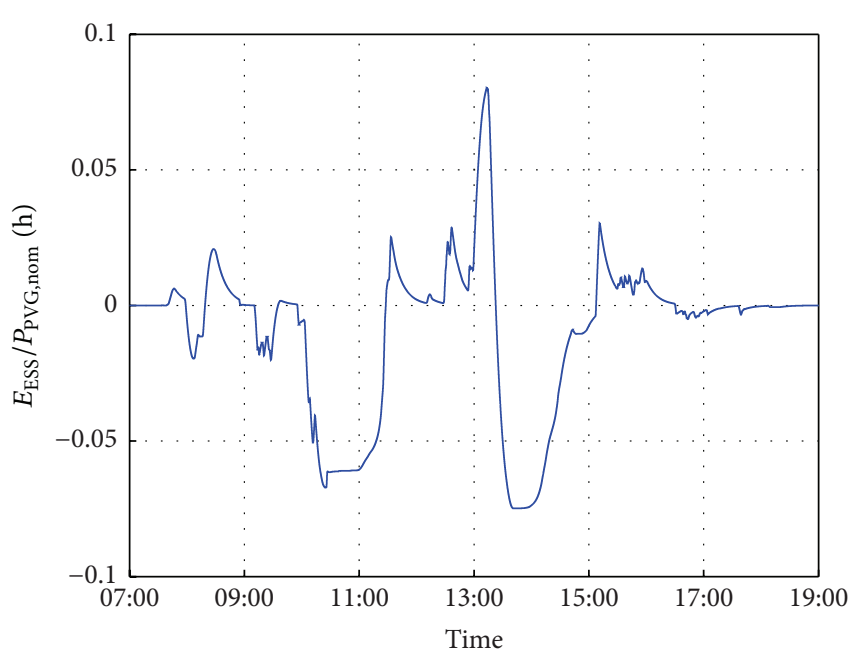

(c)

FIGURE 4: (a) Output power and ramp rate limited grid feed-in power of the $0.55 \mathrm{MWp}$ PV generator, (b) power fed to the grid by ESS, and (c) energy stored to ESS while complying to RR limit of 3\%/min on 13.08.2012. All variables have been normalized to the generator nominal power.

There are major differences in the ESS capacity requirements between the TUT and UNav results in Figure 5. The difference seems to be highest at low RR limits and decrease with increasing limit. To have a closer look on the ESS capacity requirements between the UNav and TUT systems, their relative difference is shown in Table 1 for several $R R$ limits and the two PV generator sizes. It is clearly revealed how the UNav system requires as much as $50 \%$ more ESS capacity than the TUT system when high compensation of power fluctuations is required. For the typical RR limit requirement of $10 \% / \mathrm{min}$, the difference is still $24 \%$ for the smaller PV generator but decreases down to $5 \%$ for RR limit of $30 \% / \mathrm{min}$. For the bigger generator, the difference remains high above $30 \%$ also for high RR limits. One can conclude that applying the UNav results based on the WFM
TABLE 1: The relative difference of the UNav ESS energy capacity requirements with respect to the TUT requirements for different ramp rate limits and the two sizes of PV generators.

\begin{tabular}{lcccccc}
\hline RR limit (\%/min) & 1 & 3 & 5 & 10 & 20 & 30 \\
\hline$C_{0.55 \mathrm{MWp}_{\mathrm{p}}(\%)}$ & 50 & 48 & 32 & 24 & 15 & 5 \\
$C_{2.2 \mathrm{MWp}_{\mathrm{p}}}(\%)$ & 50 & 46 & 34 & 32 & 33 & 34 \\
\hline
\end{tabular}

charging control procedure to PV power systems located in the Northern European climate could lead to drastic oversizing of ESS.

The large differences between the UNav and TUT energy storage capacity requirements are interesting, considering that the WFM charging control approach as such is logical 


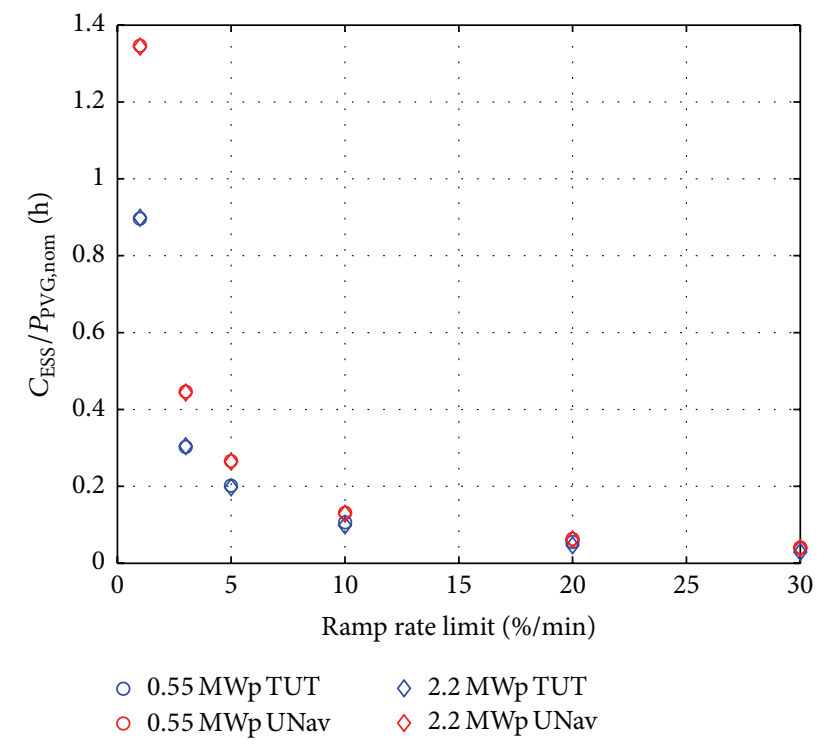

FIGURE 5: Energy capacity requirement for ESS as a function of the ramp rate limit for two sizes of PV generators located at Tampere region in Finland and Navarra region in Spain [9]. Capacities are normalized to the generators nominal power.

and the original aim of the TUT approach was to improve ESS operation mainly on the point of view of the power system operation. In order to find out the origin of these differences, the formation of the maximum absolute ESS capacity requirement of the TUT simulations during the year 2012 is shown in Figure 6 for the 0.55 MWp PV generator complying with RR limit of $3 \% / \mathrm{min}$.

From Figure 6, it is clear that the annual minimum of stored energy to ESS in the TUT system is formed entirely during a single large fluctuation, just like by applying the WFM. However, the steadily declining $P_{\text {grid }}$ ramp stops declining after reaching a level of 0.3 p.u., which is 0.2 p.u. higher than what the WFM assumes. The WFM model assumes that the power decreases exponentially from 1.0 p.u. to 0.1 p.u. during the irradiance fluctuations. The duration of an uninterrupted $P_{\text {grid }}$ ramp is the key factor in determining the minimum value of $E_{\mathrm{ESS}}$ in such a fluctuation. The WFM control assumes a ramp time of 33 minutes with RR limit of $3 \% / \mathrm{min}$, but in Figure 6 the ramp takes only about 23 minutes with the enhanced TUT control, resulting in lower discharged energy. Also fluctuations of the power fed to the grid decrease considerably when applying the TUT approach.

An example of strict power fluctuation compensation is presented in Figure 7, which displays the formation of the absolute maximum ESS energy deflection from the reference level during the year 2012 for the $0.55 \mathrm{MWp}$ PV generator complying with $\mathrm{RR}$ limit of $1 \% / \mathrm{min}$. The strict $\mathrm{RR}$ limit changes $P_{\text {grid }}$ behavior considerably from the $3 \% / \mathrm{min}$ case. $P_{\text {grid }}$ ramp descends for about 85 minutes, which is close to what the WFM also assumes. However, this time $P_{\mathrm{PVG}}$ fall is gradual, resulting in gentle slope of discharged energy. With this gentle slope, the discharged total energy of TUT is much smaller than what the WFM gives.
It is important to keep in mind that the moments presented in Figures 6 and 7 show the worst possible fluctuations experienced with the selected RR limits during the year 2012 in the Tampere area. The UNav capacity results, which have been obtained with simulation studies based on real PVG data using the WFM charge control, correlate qualitatively with our results. The WFM control is obviously quite logical providing a useable worst-case estimate for the required ESS capacity. However, the WFM seems to be too strict leading to oversizing of the ESS capacity. For optimum ESS sizing, more thorough analysis based on measurements should be used rather than assumptions based on worst-case models such as the WFM.

5.2. Maximum Power Requirements. Annual maximum ESS power requirements were also analyzed from the ESS simulations and the results can be seen in Figure 8 for the 0.55 and 2.2 MWp PV generators. The maximum power requirement decreases as the RR limit increases, but the decrease is much slower than in the case of the energy storage capacity requirement. The power capacity requirement decreases a bit faster for the 2.2 MWp generator than for the $0.55 \mathrm{MWp}$ generator and the TUT results decrease faster than the UNav results. In practice, the applied RR limit does not seem to be very critical on the point of view of ESS power capacity sizing.

The TUT ESS power requirements differ from the UNav requirements being a bit higher at low RR limits for both generator sizes and clearly lower at high RR limits. In other words, the TUT power requirements have much steeper slopes as a function of the RR limit. Reasons behind these differences were once again found by studying the formation of the TUT requirements in detail. The formation of the power requirements for the $0.55 \mathrm{MWp}$ PVG complying with RR limit of $1 \% / \mathrm{min}$ is shown in Figure 9 and for the $2.2 \mathrm{MWp}$ PVG complying with RR limit of $20 \% / \mathrm{min}$ is shown in Figure 10.

In Figure 9, a large downward $P_{\mathrm{PVG}}$ fluctuation has required a lot of compensation energy (prior to 11:30), which the SOC control is slowly trying to regain by keeping $P_{\text {grid }}$ at a low regulated constant value until 11:37 o'clock. $P_{\text {grid }}$ regulation is still ongoing due to the low RR limit of $1 \% / \mathrm{min}$ when a large upward $P_{\mathrm{PVG}}$ fluctuation begins, creating a rapidly increasing demand to charge ESS to compensate the upward ramp. Because the rise is so rapid and the starting point of $P_{\text {grid }}$ is very low, the resulting $P_{\mathrm{ESS}}$ peak is very high close to 1 p.u. (see Figure 8), which is 0.07 p.u. higher than the UNav result. Also the ESS charging is done for a long time after the ramp with almost the same power as the PV generator produces. It is also demonstrated nicely in Figure 9 how a high requirement to compensate PV power fluctuations (small RR limit) leads to a high requirement for the ESS storage capacity.

The behavior of $P_{\text {grid }}$ changes entirely when less demanding compensation of $\mathrm{PV}$ generator power fluctuations is required by applying high $\mathrm{RR}$ limits. The annual maximum ESS power requirement in Figure 10 reaches only a value of 0.65 p.u., although the generator power goes simultaneously above 1 p.u. The main reason for this is the higher RR limit but also the smoother $P_{\mathrm{PVG}}$ behavior has resulted in a higher 


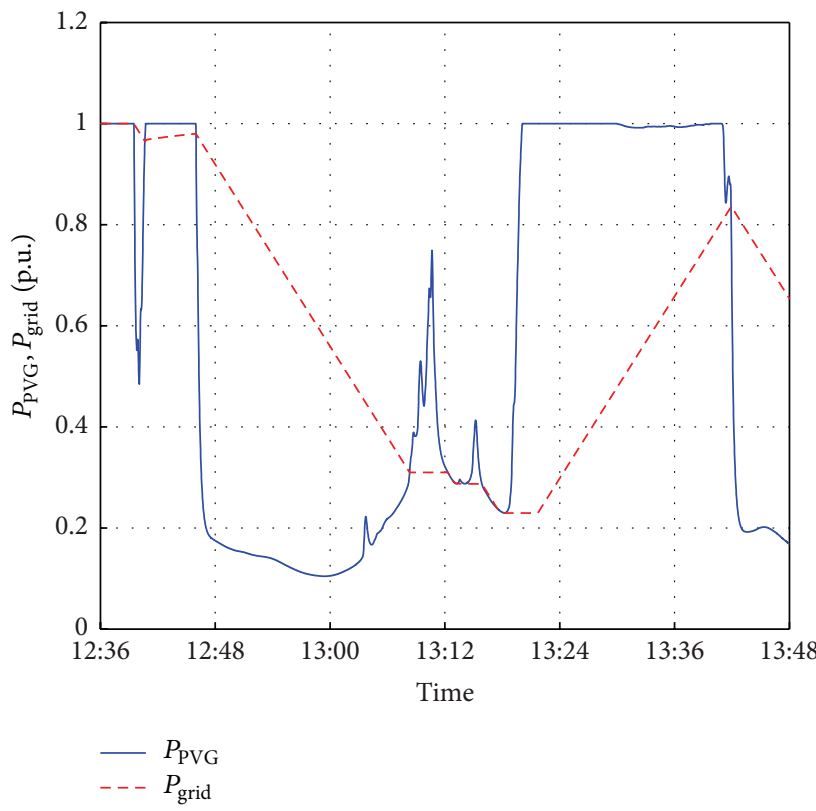

(a)

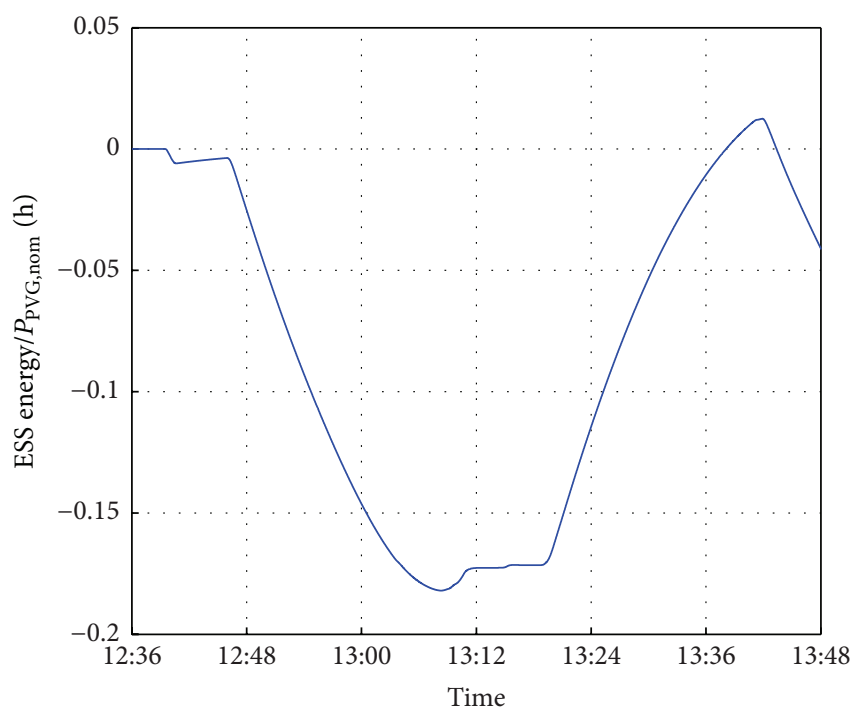

(b)

FIGURE 6: (a) 0.55 MWp PV generator and grid feed-in power and (b) the ESS energy during the formation of the minimum ESS energy with respect to the reference level causing the maximum absolute ESS capacity requirement of the TUT simulations on 09.04.2012 while complying with RR limit of 3\%/min.

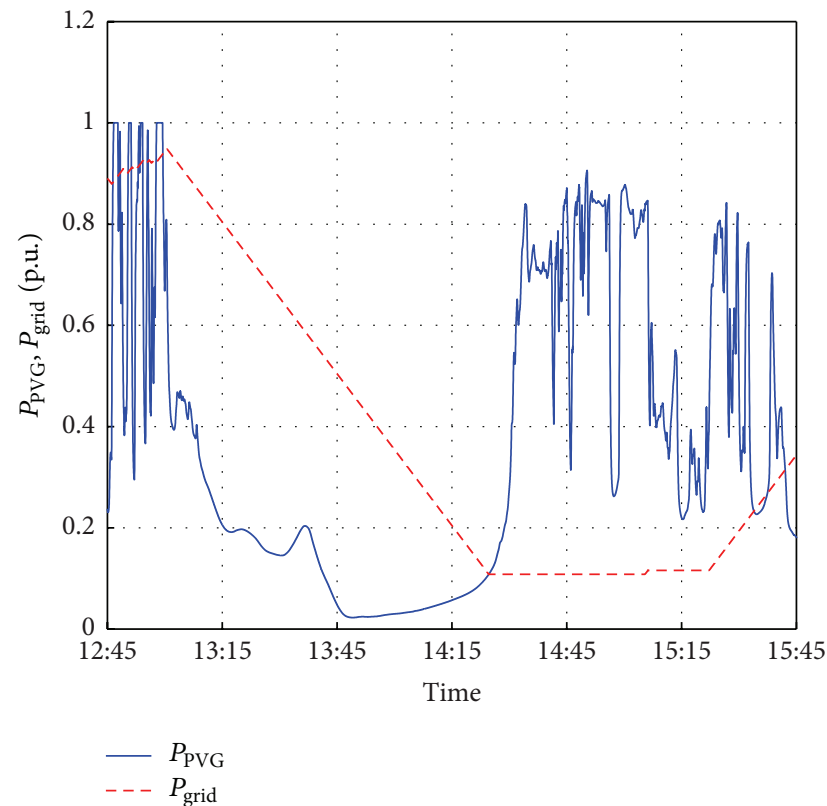

(a)

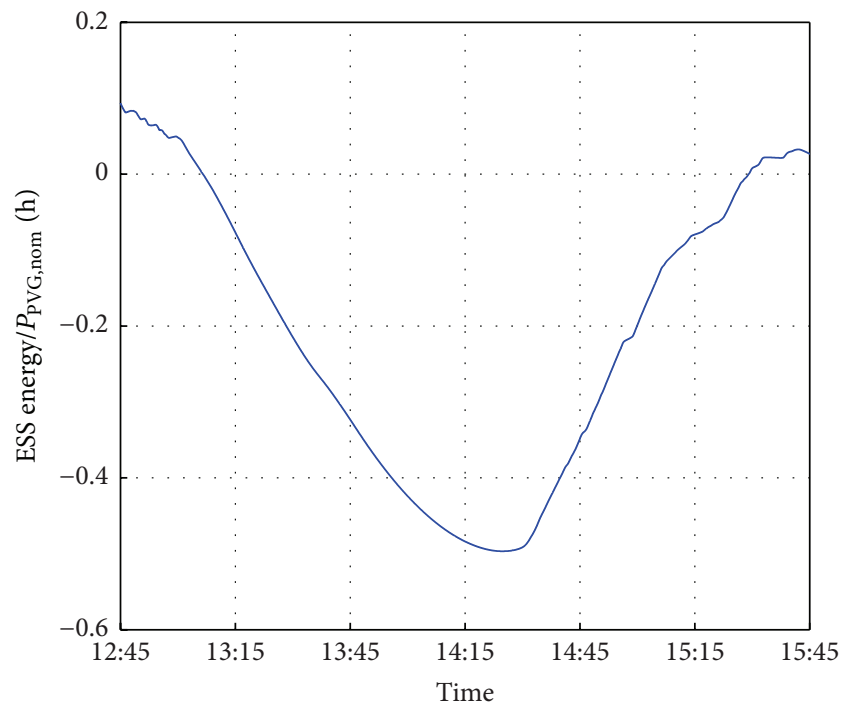

(b)

FIGURE 7: (a) 0.55 MWp PV generator and grid feed-in power and (b) the ESS energy during the formation of the minimum ESS energy with respect to the reference level causing the maximum absolute ESS capacity requirement on 27.06.2012 while complying with RR limit of $1 \% / \mathrm{min}$.

starting point for $P_{\text {grid }}$ before the fluctuation. These translate into a smaller ESS power requirement compared to what UNav results imply.

The WFM charge control assumes that $P_{\text {grid }}$ level ranges from 10 to $100 \%$ of $P_{\mathrm{PVG}, \text { nom }}$ during PVG power fluctuation.
The minimum is defined with an assumption that the midday diffuse irradiance will always yield at least $10 \%$ of the clear sky PV power generation. Also the irradiance fluctuation is assumed to be a step function leading to an exponential PVG power fluctuation, which is actually never the case with real 


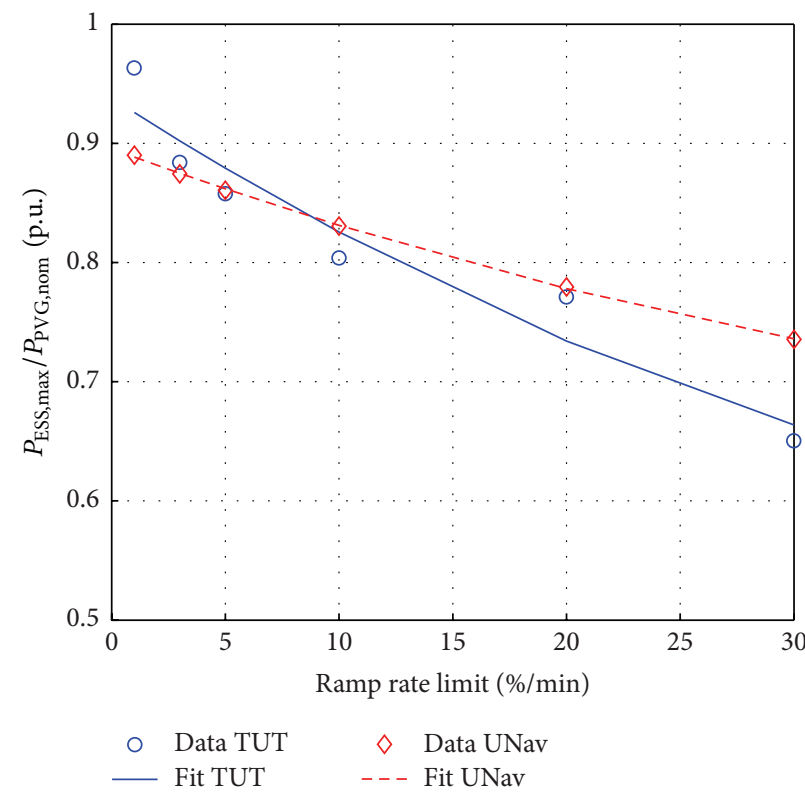

(a)

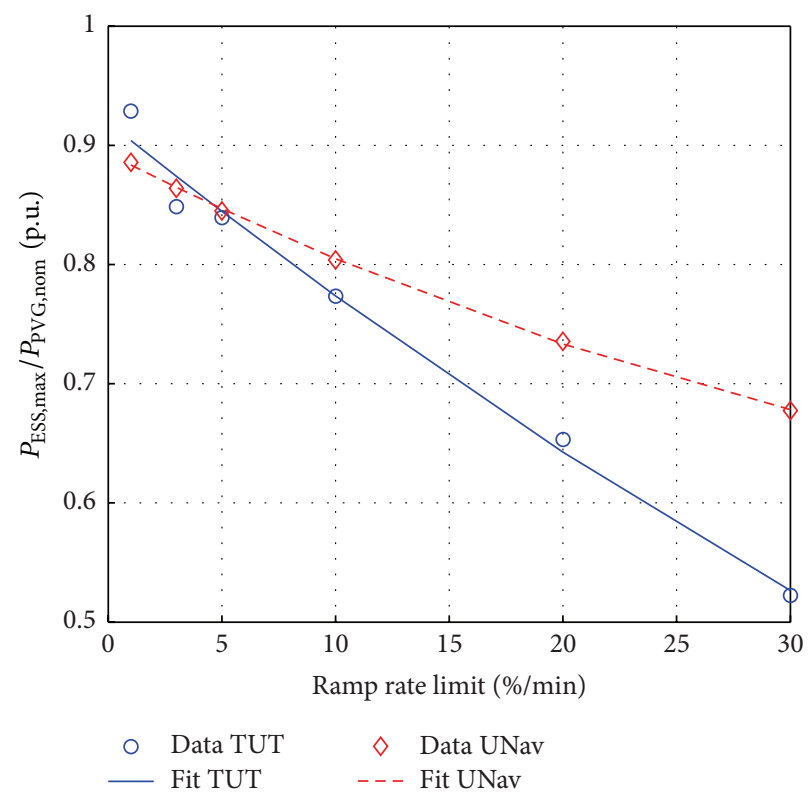

(b)

FIGURE 8: Simulated ESS maximum power requirements as functions of the ramp rate limit for (a) the $0.55 \mathrm{MWp}$ and (b) $2.2 \mathrm{MWp}$ PV generators. Values have been normalized to the generators nominal power. Overall trends have been depicted with the second-order polynomial fits. The results obtained by the researchers at the University of Navarra for corresponding PV generators located in western Spain are shown for comparison [9].

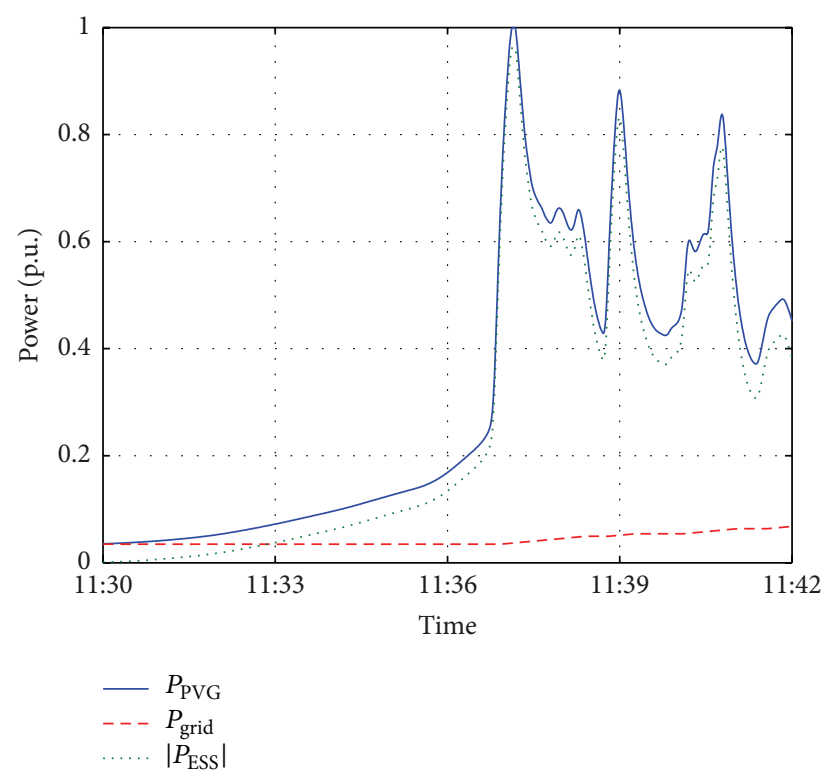

FIGURE 9: Output power of the 0.55 MWp PV generator, grid feedin power, and the absolute power of ESS during the formation of the maximum ESS power requirement on 08.09.2012 while complying with RR limit of $1 \% / \mathrm{min}$.

fluctuations caused by cloud shading. Figures 9 and 10 clearly show that these assumptions do not come true as such in real cases. Those assumptions are reasonable for obtaining a rough worst-case estimate, but they can easily lead to oversizing of ESS.

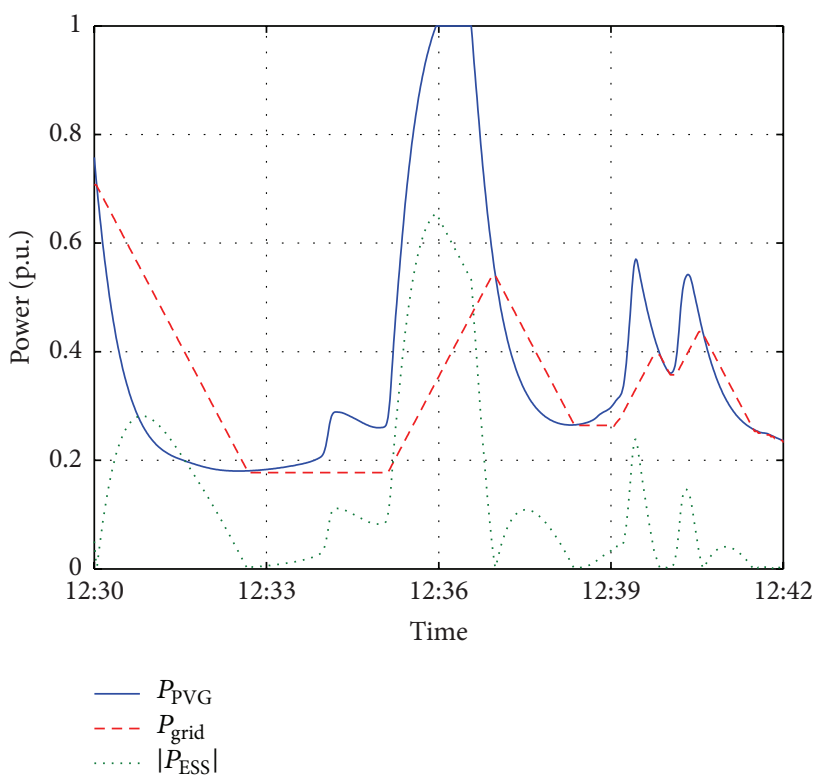

FIGURE 10: Output power of the 2.2 MWp PV generator, grid feed-in power, and the absolute power of ESS during the formation of the maximum ESS power requirement on 07.06.2012 while complying with RR limit of $20 \% / \mathrm{min}$.

5.3. Cycling Induced Storage Degradation. In addition to energy capacity and power requirements, a key sizing issue and storage technology selection factor is the determination of the fatigue that the ESS is subjected to in this application. Application induced fatigue can be measured by counting 


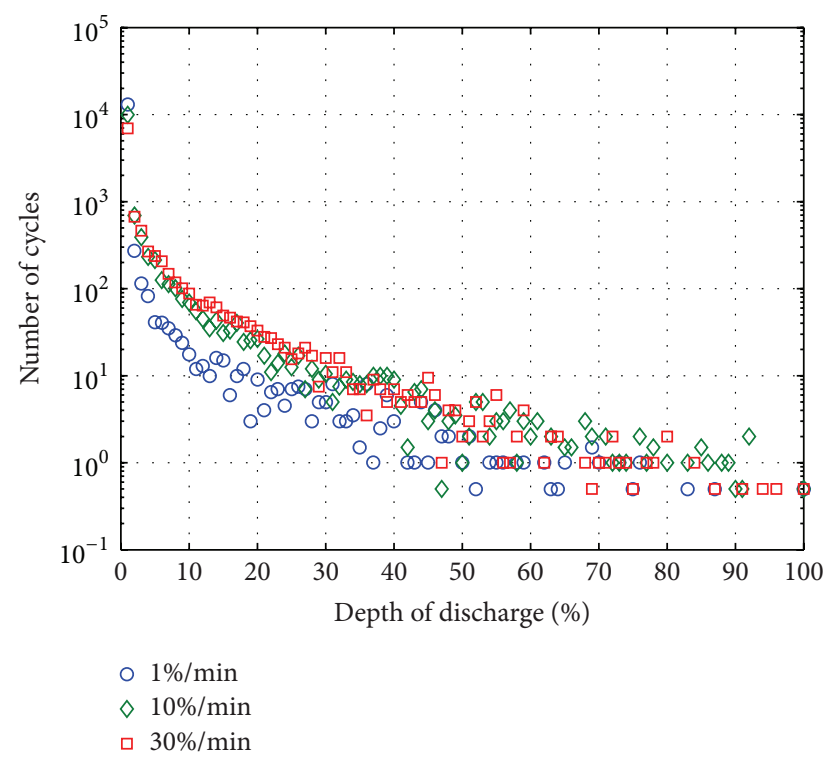

FIGURE 11: Observed number of energy storage charge-discharge cycles as a function of the depth of discharge for compensating the $0.55 \mathrm{MWp}$ PV generator using three different ramp rate limits during the year 2012. The depth of discharge is calculated with respect to the observed effective energy capacity requirements shown in Figure 5.

the number of charge-discharge cycles. With considerable cycling, the ESS can degrade so much that it no longer satisfies its design criteria and has to be replaced. For example, the amount of annual degradation compared to the expected lifetime of the PV system determines whether a specific storage technology is viable in this application or not.

In practice, cycling induced degradation can be determined by counting the number of observed ESS chargedischarge cycles $N$. ESS can be charged or discharged in random sequences with any amplitude between 1 and 100\% of its effective capacity. This amplitude is referred to as the depth of discharge (DOD). ESS can have a different cycle life depending on the DOD. Thus, cycles have to be counted separately for each degree of the DOD. In this paper, the popular rainflow-counting algorithm was used to count the cycles [16]. The cycles were counted for different cases using the two different PVG sizes and various RR limits. The number of cycles as a function of DOD can be seen in Figures 11 and 12 for the two PV generator sizes and different RR limits. It should be noted that DOD is expressed with respect to the effective energy capacity required for each case. Therefore, a higher number of small DOD cases exist when applying high RR limits than when applying low RR limits (Figure 11), which require higher maximum ESS energy capacity. Also some storage technologies might not be able to withstand deep $80-100 \%$ discharges, which should be taken into account by adjusting $E_{\mathrm{ESS}, \text { ref }}$ and $C_{\mathrm{ESS}}$ accordingly.

The number of charge-discharge cycles decreases exponentially with increasing depth of discharge for all the generator sizes and RR limits presented in Figures 11 and 12. This means that the ESS mostly compensates small fluctuations rather than large ones. The number of cycles in Figure 12

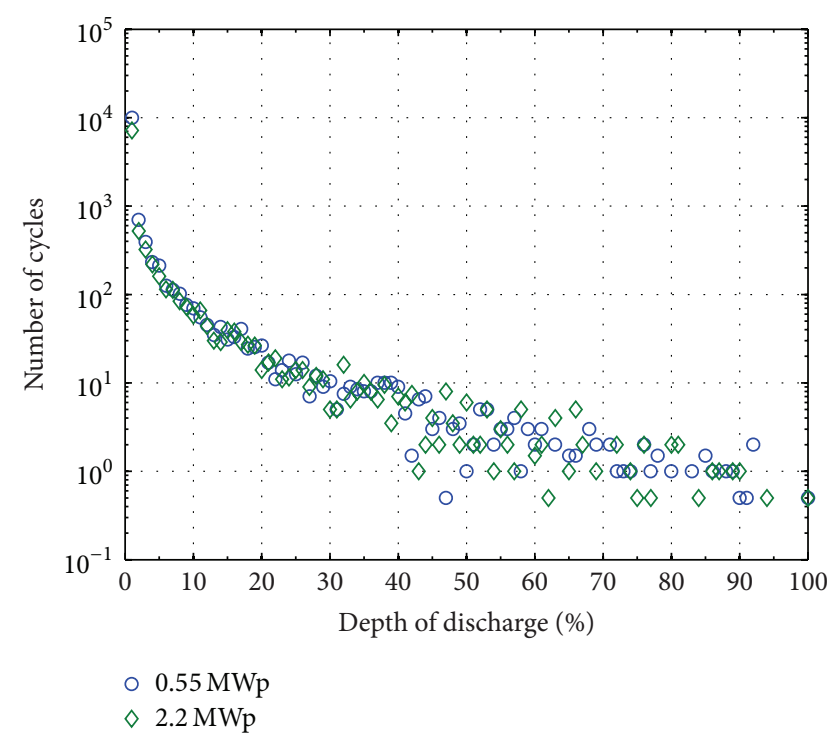

FIGURE 12: Observed number of storage charge-discharge cycles as a function of the depth of discharge for compensating two different generator sizes complying to a ramp rate limit of $10 \% / \mathrm{min}$ during the year 2012. The depth of discharge is calculated with respect to the observed effective energy capacity requirements shown in Figure 5.

indicates that the PV generator size has no significant effect on overall cycling.

The results from the cycle counting as such do not reveal much, and no trends are visible either. To obtain a more precise indicator for ESS degradation, a comparison to the maximum cycling tolerance of ESS is necessary. This means that a specific ESS needs to be selected. In this paper, as an example, a degradation study is done for ESS containing lithium-titanate batteries from Altairnano [17]. The cycle life curve, that is, the maximum amount of tolerable cycles $N_{\mathrm{ESS}, \text { max }}^{\mathrm{DOD}}$ for each DOD value, of this battery is depicted in Figure 13.

The maximum number of cycles as a function of DOD presented in Figure 13 is used as a reference which the observed number of cycles $N_{\text {obs }}^{\text {DOD }}$ for each DOD value has been compared to (Figures 11 and 12). The overall annual degradation is obtained as the sum of individual comparisons over all degrees of DOD as follows:

$$
C_{\mathrm{deg}}=\sum_{\mathrm{DOD}=1 \%}^{100 \%} \frac{N_{\mathrm{obs}}^{\mathrm{DOD}}}{N_{\mathrm{ESS}, \max }^{\mathrm{DOD}}} \cdot 100 \%
$$

By using (4) and the previously presented annual cycle counts, the cycling induced degradation of an Altairnano ESS was calculated. The results for the two PV generators and different RR limits are presented in Table 2. The main observation is that the amount of degradation is below $0.5 \%$ in a year in almost all cases. A conclusion from this is that storage technologies do not cause insuperable fatigue obstacles on ESS application, although the low level of degradation in Table 2 is due to the selection of a highly cycledurable battery technology. 
TABLE 2: Cycling induced degradation of an Altairnano battery energy storage system compensating the power fluctuations of two different PV generators with different ramp rate limits located in Northern European climate during the year 2012.

\begin{tabular}{lcccccc}
\hline RR limit (\%/min) & 1 & 2 & 5 & 10 & 20 & 30 \\
\hline$C_{\text {deg, } 0.55 \mathrm{MWp}}(\%)$ & 0.22 & 0.31 & 0.36 & 0.47 & 0.53 & 0.47 \\
$C_{\text {deg, } 2.2 \mathrm{MWp}}(\%)$ & 0.20 & 0.27 & 0.31 & 0.42 & 0.47 & 0.42 \\
\hline
\end{tabular}

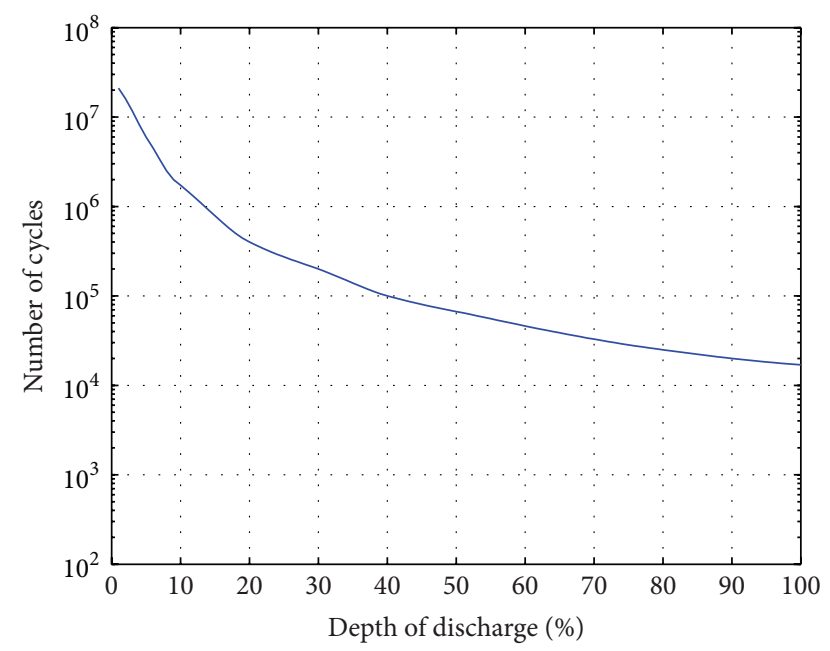

FIgURE 13: Maximum number of cycles as a function of DOD for an Altairnano lithium-titanate battery adapted from [17] $\left(\text { Altairnano }^{(}\right)^{\text {. }}$.

Degradation increases with increasing RR limit up to the $20 \% / \mathrm{min}$ and after that seems to reduce. This is in line with the earlier observed smaller number of ESS cycles when applying a small RR limit instead of high RR limits shown in Figure 11. Degradation seems to decrease slightly also with increasing PV generator size, which is in line with the small differences of the number of cycles between the two PVG sizes in Figure 12.

The amount of degradation is of the same order of magnitude with similar degradation studies conducted in Southern Europe with a Li-ion battery technology [11]. Due to differences in determining the ESS requirements, the effective DOD ranges, and the selected ESS technologies, a more thorough comparison is not meaningful. An overall conclusion from this degradation study is that, by selecting the right technology for ESS, PV power fluctuations can be compensated irrespective of the required RR limit without unnecessarily high cycling degradation.

\section{Conclusions}

Requirements for sizing an energy storage system to compensate PV generator's power fluctuations with various ramp rate limits have been determined in this paper. The study utilized irradiance and PV module back plate temperature measurements performed by the Tampere University of Technology solar PV power research plant located in Tampere,
Finland. A first-order low-pass filter method was applied to estimate spatial smoothing of irradiance over different PV generator areas. PV generator output power was then modelled using the smoothed wide area irradiance and PV module temperature as inputs.

The model was further used to provide scalable and general PV generator behavior as an input for energy storage system simulations. The modelled PV generator power was fed through the ramp rate limiter to calculate the desired limit compliant grid feed-in power. The required energy storage power was then determined as the difference between the grid feed-in and the PV generator power. In addition, an enhanced state of charge control strategy was developed and applied to decrease the magnitude of the grid feed-in power fluctuations. A full year of irradiance and temperature measurements with a sampling frequency of $10 \mathrm{~Hz}$ were then used to simulate the operation of the energy storage system for 0.55 and 2.2 MWp PV generators using different ramp rate limits.

Effective energy storage capacity, power, and chargedischarge-cycling were determined from the simulations. The required maximum energy storage capacity was found to be directly proportional to the PV generator nominal power and to decrease exponentially with decreasing requirements to compensate PV generator power fluctuations, that is, with increasing ramp rate limit. The required maximum power of the energy storage system decreases linearly with increasing ramp rate limit. Additionally, it decreases slightly with increasing PV generator size, when normalized to the generator nominal power. Cycling induced degradation of a selected commercial battery technology was shown to be below $0.5 \%$ per year for both PV generator sizes and for almost all ramp rate limits. Degradation increases with increasing ramp rate limit up to $20 \% / \mathrm{min}$ and then decreases. An overall conclusion is that storage technologies do not cause overwhelming obstacles on ESS application for compensation of fast PV power fluctuations.

The sizing requirements presented in this paper were also compared to requirements obtained in similar studies done in a Southern European climate in Spain. The comparison showed significant differences in energy capacity and maximum power requirements between the two studies. Most notably, the capacity requirements for the energy storage system were up to $50 \%$ higher in the reference study than in this study when high compensation was required. The large differences are mainly achieved by the enhanced state of charge control strategy applied in our study, but also the climatic differences may have caused some differences. The differences translate directly into smaller ESS sizing requirements and better operation of the storage system. Therefore, in order to determine optimum energy storage systems to limit PV generator power fluctuations, advanced control strategies utilizing local measurements should be used for system sizing.

\section{Competing Interests}

The authors declare that they have no competing interests. 


\section{References}

[1] J. Marcos, L. Marroyo, E. Lorenzo, and M. García, "Smoothing of PV power fluctuations by geographical dispersion," Progress in Photovoltaics: Research and Applications, vol. 20, no. 2, pp. 226-237, 2012.

[2] R. van Haaren, M. Morjaria, and V. Fthenakis, "Empirical assessment of short-term variability from utility-scale solar PV plants," Progress in Photovoltaics: Research and Applications, vol. 22, no. 5, pp. 548-559, 2014.

[3] V. Gevorgian and S. Booth, "Review of PREPA Technical Requirements for Interconnecting Wind and Solar Generation," 2013, http://www.nrel.gov/docs/fy14osti/57089.pdf.

[4] S. Rahman and K.-S. Tam, "A feasibility study of photovoltaicfuel cell hybrid energy system," IEEE Transactions on Energy Conversion, vol. 3, no. 1, pp. 50-55, 1988.

[5] B. Yang, Y. Makarov, J. Desteese et al., "On the use of energy storage technologies for regulation services in electric power systems with significant penetration of wind energy," in Proceedings of the 5th International Conference on the European Electricity Market (EEM '08), pp. 1-6, Lisboa, Portugal, May 2008.

[6] N. Kakimoto, H. Satoh, S. Takayama, and K. Nakamura, "Ramprate control of photovoltaic generator with electric double-layer capacitor," IEEE Transactions on Energy Conversion, vol. 24, no. 2, pp. 465-473, 2009.

[7] T. D. Hund, S. Gonzalez, and K. Barrett, "Grid-tied PV system energy smoothing," in Proceedings of the 35th IEEE Photovoltaic Specialists Conference (PVSC '10), pp. 2762-2766, IEEE, Honolulu, Hawaii, USA, June 2010.

[8] G. Karmiris and T. Tengnér, "Control method evaluation for battery energy storage system utilized in renewable smoothing," in Proceedings of the 39th Annual Conference of the IEEE Industrial Electronics Society (IECON '13), pp. 1566-1570, Vienna, Austria, November 2013.

[9] J. Marcos, O. Storkël, L. Marroyo, M. Garcia, and E. Lorenzo, "Storage requirements for PV power ramp-rate control," Solar Energy, vol. 99, pp. 28-35, 2014.

[10] I. de la Parra, J. Marcos, M. García, and L. Marroyo, "Control strategies to use the minimum energy storage requirement for PV power ramp-rate control," Solar Energy, vol. 111, pp. 332-343, 2015.

[11] J. Marcos, I. de La Parra, M. García, and L. Marroyo, "Control strategies to smooth short-term power fluctuations in large photovoltaic plants using battery storage systems," Energies, vol. 7, no. 10, pp. 6593-6619, 2014.

[12] C. Rus-Casas, J. D. Aguilar, P. Rodrigo, F. Almonacid, and P. J. Pérez-Higueras, "Classification of methods for annual energy harvesting calculations of photovoltaic generators," Energy Conversion and Management, vol. 78, pp. 527-536, 2014.

[13] D. Torres Lobera, A. Mäki, J. Huusari, K. Lappalainen, T. Suntio, and S. Valkealahti, "Operation of TUT solar PV power station research plant under partial shading caused by snow and buildings," International Journal of Photoenergy, vol. 2013, Article ID 837310, 13 pages, 2013.

[14] J. Marcos, L. Marroyo, E. Lorenzo, D. Alvira, and E. Izco, "From irradiance to output power fluctuations: the PV plant as a low pass filter," Progress in Photovoltaics: Research and Applications, vol. 19, no. 5, pp. 505-510, 2011.

[15] J. Schnabel and S. Valkealahti, "Compensation of PV generator output power fluctuations with energy storage systems," in Proceedings of the 31st European Photovoltaic Solar Energy
Conference and Exhibition, Hamburg, Germany, September 2015.

[16] M. Chawla, R. Naik, R. Burra, and H. Wiegman, "Utility energy storage life degradation estimation method," in Proceedings of the IEEE Conference on Innovative Technologies for an Efficient and Reliable Electricity Supply (CITRES '10), pp. 302-308, IEEE, Waltham, Mass, USA, September 2010.

[17] Altair Nanotechnologies Inc, PowerRack@ Product Overview, 2015, http://www.altairnano.com/products/powerrack/. 

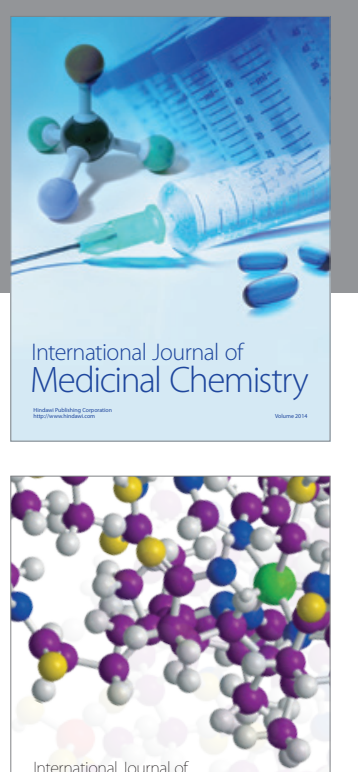

Carbohydrate Chemistry

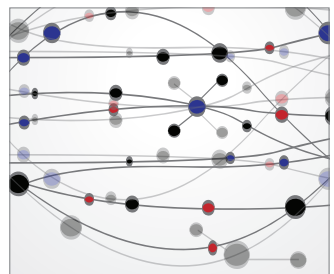

The Scientific World Journal
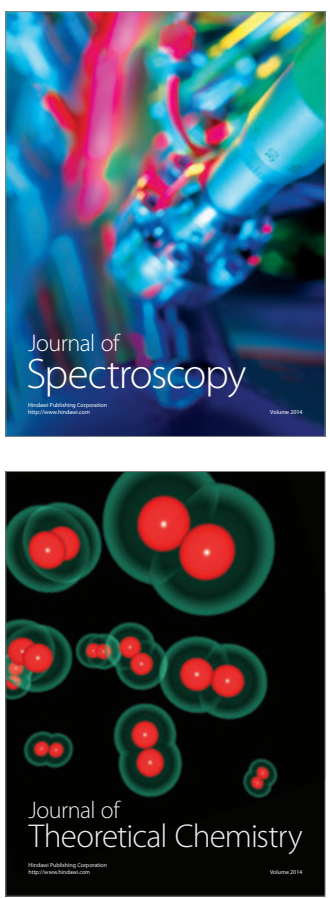
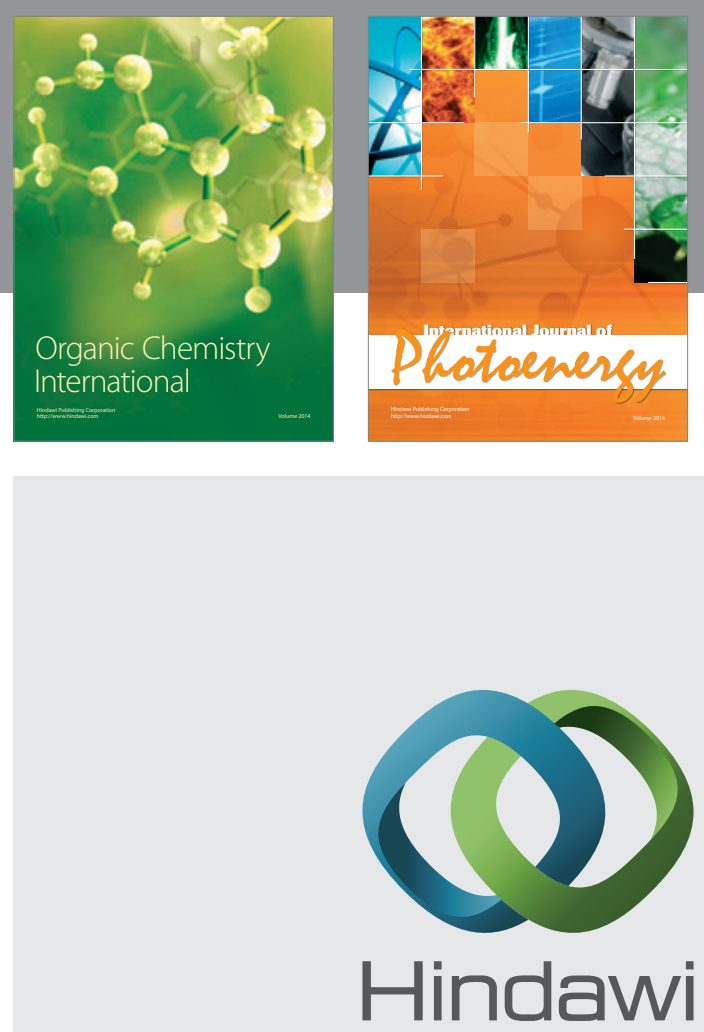

Submit your manuscripts at

http://www.hindawi.com

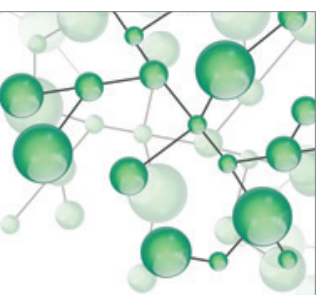

International Journal of

Inorganic Chemistry

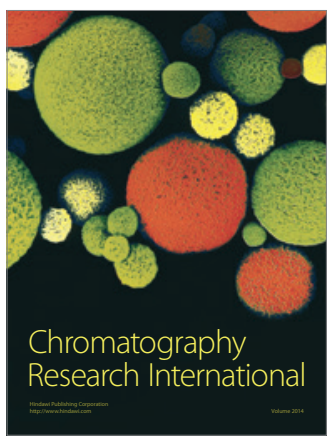

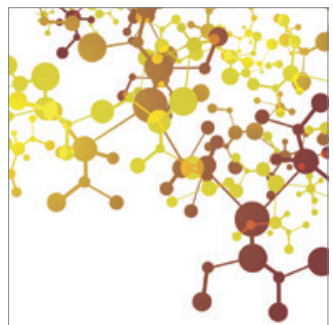

Applied Chemistry
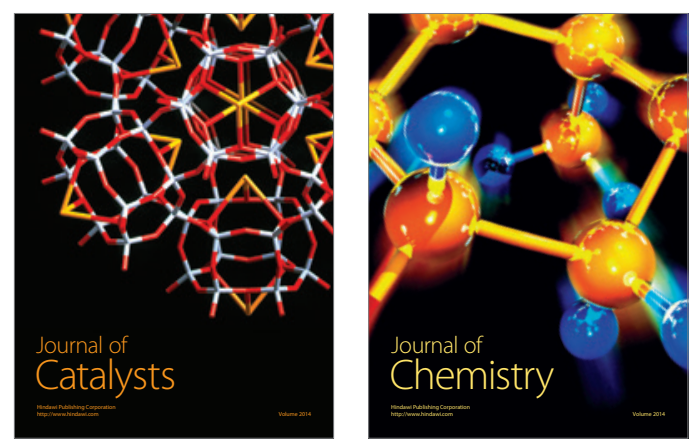
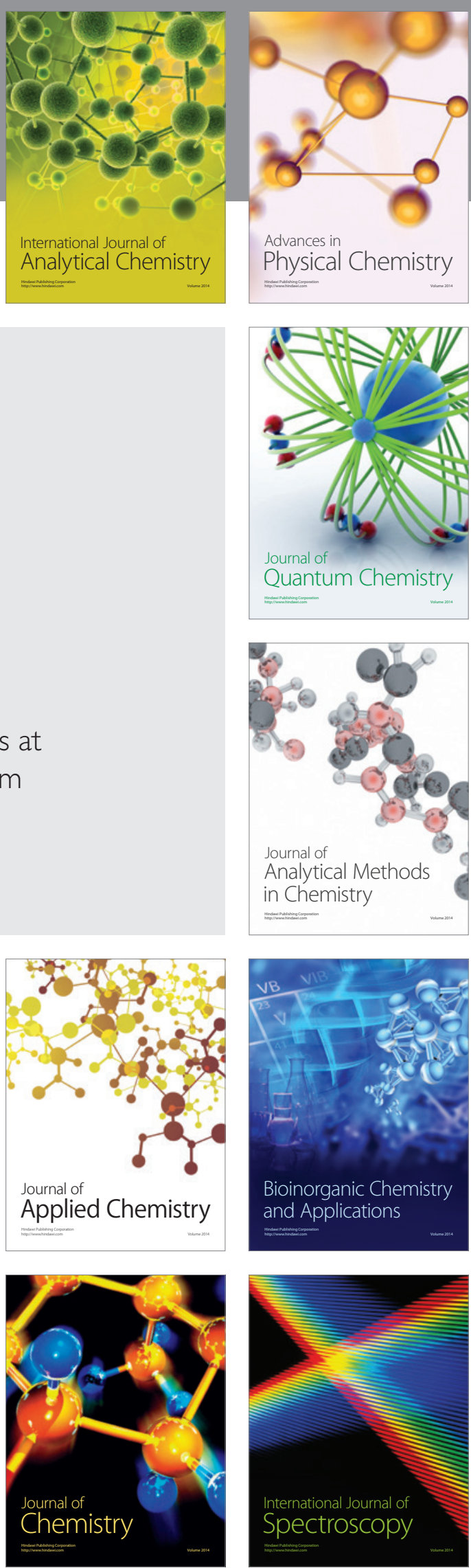